Math. Zeitschr. 105, 59-61 (1968)

\title{
A Note on a Problem of D. Pompeiu
}

\author{
L. Brown*, F. Schnitzer and A. L. ShIELDS * \\ Received January 8, 1968
}

\section{Introduction}

As PoMpeIu's problem-named after the late Rumanian mathematician DimITRIE POMPEIU - we shall denote the following question. Let $B$ be a bounded region of the $x y$-plane and let $T$ be the set of the Euclidean transformations of the plane. Suppose that $f(x, y)$ is a function of the real variables $x$ and $y$, continuous in the whole $x y$-plane and satisfying

$$
\iint_{\tau(B)} f(x, y) d x d y=0, \quad \tau \in T_{1}
$$

for some subset $T_{1} \subset T$. Does this imply that $f(x, y) \equiv 0$ ? PoMPEIU thought that if $B$ is a disc and if $T_{1}=T$, then $f(x, y) \equiv 0$ [6]. It was noted later that the result is not correct in this form. The function $f(x, y)=\sin a x$, for a suitable choice of $a$, provides a counter example. This example shows that even if one assumes that $f$ is bounded, the conclusion $f=0$ need not hold.

PompeIU proved in [7] the following result: if $B$ denotes a square and if $f(x, y)$ is a function of the real variables $x$ and $y$, continuous in the whole $x y$-plane and having a limit as $x^{2}+y^{2} \rightarrow \infty$, then (1) holds for all $\tau \in T$ if, and only if, $f(x, y) \equiv 0$. The Bulgarian mathematician Christo Christov established in [1] and [2] PompeIU's result (and similar results if $B$ is a triangle or a parallelogram) without the condition of the existence of $\lim f(x, y)$. See [3] for further references.

It is the purpose of this note to present a result which can be considered as a contribution to the study of PoMPEIU's problem. Our result is more special than Christov's in that we assume that $f(x, y)$ has a limit as $x^{2}+y^{2} \rightarrow \infty$. On the other hand our result is more general in that we use only the translations rather than all Euclidean transformations. Also, we replace the Lebesque area measure $d x d y$ on a square by any product measure $d \mu(x) d \nu(y)$ where $\mu, v$ are arbitrary complex-valued measures of compact support. The method applied uses a few facts from the theory of mean periodic functions in one variable as, for example, presented in the lecture notes by J. P. KaHANE [4].

\section{Background Material}

A complex-valued continuous function $f$ defined on the real line is said to be mean periodic if there is a complex-valued measure $\mu$, not identically zero, of

$\star$ The research of these authors was partially supported by the National Science Foundation. 
compact support, such that

$$
\int f(x+y) d \mu(y)=0 \quad(-\infty<x<\infty) .
$$

Next, we shall define almost periodic functions (in the sense of BoHR). We say, given any $\varepsilon>0$, that the number $\tau>0$ is an almost period corresponding to $\varepsilon$ for the complex-valued continuous function $f(x)$ if $\left|f_{\tau}(x)-f(x)\right|<\varepsilon$ for all $x$, where $f_{\tau}$ is the translate of $f: f_{\tau}(x)=f(x+\tau)$. A set $M$ of reals will be called relatively dense if there exists an $L>0$ such that in any interval of length $L$ there lies at least one point of M. HARALD BoHR then defined [5]: A function $f(x)$ complex-valued and continuous, is almost periodic if, for every $\varepsilon>0$, the almost periods of $f$ form a relatively dense set.

For the proof of our theorem we need the following facts about mean periodic and almost periodic functions.

Theorem A (Kahane [4]). A uniformly continuous, bounded mean periodic function is almost periodic (in the sense of $\mathrm{BOHR}$ ).

Lemma A. An almost periodic function $f(x)$ for which $\lim _{x \rightarrow \infty} f(x)$ exists is necessarily a constant.

Theorem A lies quite deep and is difficult to prove; on the other hand, Lemma $\mathrm{A}$ is trivial.

\section{Main Result}

Theorem. Let $\mu$ and $v$ be arbitrary complex-valued measures of compact support on the real line (with neither of them being the zero measure). Let $f(x, y)$ be continuous in the plane and satisfy

$$
\begin{gathered}
\lim f(x, y) \text { exists }\left(x^{2}+y^{2} \rightarrow \infty\right), \\
\iint f(x+s, y+t) d \mu(x) d v(y)=0, \quad-\infty<s, t<\infty .
\end{gathered}
$$

Then $f=$ constant. If further

$$
\int d \mu \neq 0 \text { and } \int d v \neq 0
$$

then $f=0$.

Proof. Let $c=\lim f(x, y)\left(x^{2}+y^{2} \rightarrow \infty\right)$. First note that if (3) does not hold then $f-c$ satisfies (1) and (2). On the other hand, if (3) does hold then $c=0$, since $\iint c d \mu d \nu=0$ by (2).

Thus in any case we may assume without loss of generality that $c=0$.

For fixed $s$ consider the function $\phi(y)=\int f(s+x, y) d \mu(x)$. We have:

$$
\int \phi(y+t) d v(y)=0 \quad(-\infty<t<\infty)
$$

and so $\phi(y)$ is mean periodic. Furthermore, $\phi$ is continuous and

$$
\lim \phi(y)=0 \quad(|y| \rightarrow \infty),
$$

and so $\phi$ is bounded and uniformly continuous. By Theorem A and Lemma A, $\phi=0$. 
Thus we have shown that for each fixed $s$ and for all $y$ we have

$$
\int f(s+x, y) d \mu(x)=0 .
$$

For fixed $y$, let $g(x)=f(x, y)$. The above equation tells us that $g$ is mean periodic. Also, $\lim g(x)=0(|x| \rightarrow \infty)$ and so, just as in the previous paragraph, $g=0$. But this says that for each $y$ the function $f(x, y)=0$ for all $x$, i.e., $f=0$, which completes the proof.

The fact that we had a product measure enabled us to make use of the theory of mean periodic function in one variable for solving our variant of PoMPEIU's problem. The problem of a general measure with compact support in the plane remains open.

\section{References}

1. Christov, C.: Sur un problème de M. Pompeiu. Mathematica Timisoara 23, 103-107 (1948).

2. - On the generalized integral equation of D. Pompeiu. Annaire de l'Université de Sofia Faculté des Sciences, Livre 1. 45, 177-178 (1948-49).

3. DeMar, R. F., and P. J. Davis: A complex Pompeiu problem. Duke Math. J. 33, 91-101 (1966).

4. KaHANE, J. P.: Lectures on mean periodic functions. Tata Institute of Fundamental Research. Bombay 1956.

5. MAAK, W.: Fastperiodische Funktionen. Grundlehren der Mathem. Wissenschaften, Bd. LXI. Berlin-Göttingen-Heidelberg: Springer 1950.

6. PoMPIEU, D.: Sur une propriété des fonctions continues dépendent de plusieur variables. Bulletin des Sciences Mathématique 53, 328-332 (1929).

7. - Sur une propriété intégrale des fonctions de deux variables réeles. Bulletin des Science Académie Royale de Belgique 15, 265-269 (1929).

Prof. Allen L. ShIELDS

Dept. of Mathematics

University of Michigan

Ann Arbor, Michigan 48104
Prof. LeON BRown

Prof. Franz SchNitzer

Dept. of Mathematics

Wayne State University

Detroit, Mich, 48202, USA 